\title{
Maria Piok, Ulrike Tanzer und Kyra Waldner (Hg.), Marie von Ebner-Eschenbach. Schriftstellerin zwischen den Welten
}

Marc Lacheny

\section{(2) OpenEdition}

\section{Journals}

Édition électronique

URL : http://journals.openedition.org/austriaca/469

DOI : 10.4000/austriaca.469

ISSN : 2729-0603

\section{Éditeur}

Presses universitaires de Rouen et du Havre

\section{Édition imprimée}

Date de publication : 1 décembre 2018

Pagination : 279-283

ISBN : 979-10-240-1354-1

ISSN : 0396-4590

\section{Référence électronique}

Marc Lacheny, « Maria Piok, Ulrike Tanzer und Kyra Waldner (Hg.), Marie von Ebner-Eschenbach. Schriftstellerin zwischen den Welten », Austriaca [En ligne], 87 | 2018, mis en ligne le 01 mars 2020, consulté le 28 janvier 2021. URL : http://journals.openedition.org/austriaca/469 ; DOI : https://doi.org/ 10.4000/austriaca.469

Ce document a été généré automatiquement le 28 janvier 2021.

Austriaca. Cahiers universitaires d'information sur l'Autriche 


\title{
Maria Piok, Ulrike Tanzer und Kyra Waldner (Hg.), Marie von Ebner- Eschenbach. Schriftstellerin zwischen den Welten
}

\author{
Marc Lacheny
}

\section{RÉFÉRENCE}

Maria Piok, Ulrike Tanzer und Kyra Waldner (Hg.), Marie von Ebner-Eschenbach.

Schriftstellerin zwischen den Welten (=Innsbrucker Beiträge zur Kulturwissenschaft. Germanistische Reihe. Bd. 90), Innsbruck, innsbruck university press, 2018, 236 p., ISBN : 978-3-901064-53-1, $38 €$.

1 Marie von Ebner-Eschenbach (1830-1916) s'est imposée comme auteure à une époque de bouleversements politiques, intellectuels et culturels majeurs : se frayant un chemin entre des conceptions et des valeurs traditionnelles d'un côté et sa quête d'évasion par la littérature de l'autre, elle a produit une œuvre littéraire abondante qui interroge à plusieurs niveaux les changements sociopolitiques de son temps sur la voie de la modernité. Les contributions réunies par Maria Piok, Ulrike Tanzer et Kyra Waldner dans le présent volume, fruit d'un colloque organisé en mars 2016 par la Wienbibliothek im Rathaus en coopération avec le Brenner-Archiv de l'université d'Innsbruck pour le centième anniversaire du décès de l'auteure, inscrivent précisément la vie et l'œuvre de Marie von Ebner-Eschenbach dans les contextes les plus divers, permettant ainsi de dresser le portrait, à la fois varié et complexe, d'une auteure qui semble se dérober comme "Schriftstellerin zwischen den Welten » - à toutes les étiquettes que l'on a cherché à apposer sur son œuvre comme sur sa personne.

2 Après la publication par Walter Hettche et Ulrike Tanzer d'un échantillon de textes poétiques de Marie von Ebner-Eschenbach tirés de Aus einem Liederbuche (p. 19-27), dans 
lesquels l'influence du lyrisme de Heine se fait sentir, un certain nombre de contributions portent sur l'inscription de l'œuvre de Marie von Ebner-Eschenbach dans le contexte de son époque, dont les changements trouvent écho dans sa production littéraire. Evelyne Polt-Heinzl s'intéresse ainsi aux points de contact entre Marie von Ebner-Eschenbach et les auteurs de la "Jeune Vienne " (Jung Wien), avec lesquels elle partagea un espace non seulement géographique, mais encore intellectuel, fait de bouleversements liés à l'époque de transition entre Ringstraßenzeit et modernité viennoise : outre leurs échanges épistolaires, ces auteurs fréquentaient les mêmes lieux, les mêmes concerts, les mêmes théâtres, ils publiaient dans les mêmes journaux, revues et maisons d'édition; l'auteure montre par ailleurs que de nombreux parallèles thématiques avec les auteurs du groupe Jung Wien, surtout avec Schnitzler, sont frappants, malgré d'indéniables différences dans le traitement des sujets: crise de l'individu confronté aux processus de modernisation sociale, éclatement du rapport traditionnel entre les sexes, double morale dominante. L'article de Madgalena Stieb, qui a pour objet l'impact de la photographie sur la littérature à l'exemple des nouvelles tardives de Ferdinand von Saar, paraît en revanche moins bien relié au reste du recueil - même si Marie von Ebner-Eschenbach se fait l'écho de ce nouveau médium dans son premier roman, Lotti, die Uhrmacherin (1880).

3 La plupart des contributions montrent la complexité de classer l'œuvre de Marie von Ebner-Eschenbach dans une catégorie spécifique, notamment celle d'un réalisme axé sur les notions d'harmonie et d'idylle (voir le cliché récurrent de la « Dichterin der Güte »). Pour affiner le propos, l'accent est ainsi mis sur des comparaisons avec d'autres auteurs et sur une relecture serrée de l'œuvre prise dans ces réseaux. Une question centrale ici débattue est celle de savoir dans quelle mesure Marie von EbnerEschenbach rompt avec un certain horizon d'attente conventionnel lié en particulier aux conditions de possibilité d'une écriture féminine: c'est ce que montre Peter $\mathrm{C}$. Pfeiffer, s'intéressant au début et à la fin des textes de Marie von Ebner-Eschenbach, dans son analyse du jeu de l'auteure avec les formes et les structures littéraires (tragédie historique, comédie, diverses formes de la nouvelle, poèmes), jeu par lequel elle interroge - voire dissout parfois - les frontières traditionnelles entre les genres. Cette rupture partielle avec les attentes conventionnelles liées aux genres peut également prendre la forme de projections fantasmées d'évasions et de pertes de contrôle que Daniela Strigl étudie à l'exemple des multiples scènes d'équitation, à la symbolique souvent sexuelle, qui traversent l'œuvre de Marie von Ebner-Eschenbach (Unsühnbar, Komtesse Muschi, Komtesse Paula, Die Freiherren von Gemperlein, Božena) et qui, là encore, démontent le cliché de la femme honorable en accord avec les conventions de son temps. Dans une contribution aussi courte que lumineuse, Eda Segarra se penche quant à elle sur diverses représentations de personnages enfantins dans la tradition narrative $\mathrm{du} \mathrm{XIX}^{\mathrm{e}}$ siècle, une tradition dans laquelle s'inscrit Marie von EbnerEschenbach, et parvient à la conclusion suivante: «Das ungeschundene oder ungeliebte Kind als Opfer der Gesellschaft ist ein durchgehendes Motiv der Erzählkunst der Autorin » (p. 89). Marie Luise Wandruszka montre ce qu'Ebner-Eschenbach, surtout dans Glaubenslos? et Das Gemeindekind, doit à Spinoza (l'opposition entre agir et souffrir) dans son approche du « réalisme politique». Dans un contexte d'émancipation progressive de la femme dans son rôle familial et social, Milan Tvrdík rapproche, enfin, le réalisme de Marie von Ebner-Eschenbach de celui de l'auteure tchèque Karolina Světlá (1830-1899). 
Plusieurs autres contributions s'appliquent à cerner plus spécifiquement le réseau littéraire de Marie von Ebner-Eschenbach. Deux articles portent sur la correspondance, restée longtemps inédite, entre Marie von Ebner-Eschenbach et Josephine von Knorr (1827-1908), une poétesse aujourd'hui largement oubliée; Irene Fußl et Lina Maria Zangerl montrent, à l'appui de cette très riche correspondance, les difficultés souvent insurmontables auxquelles l'auteure eut à faire face dans son rôle de soutien et de bienfaitrice de membres de sa famille: bien plus qu'une forme convenue d'échange entre amies, la lettre devient ici le substitut vital d'un espace de liberté dans lequel Marie von Ebner-Eschenbach parvint peu à peu à esquisser (Lina Maria Zangerl parle à juste titre d'Autorschaftsentwürfe), puis à développer pleinement son image d'auteure, en prenant pour modèles Betty Paoli et Annette von Droste-Hülshoff, mais aussi, sur un ton plus badin, Schiller et Goethe. Karin S. Wozonig se concentre précisément sur le rôle central joué par Betty Paoli (1814-1894) en faveur de Marie von Ebner-Eschenbach, notamment en qualité de conseillère littéraire et de "première critique (erste Rezensentin) de son œuvre. Walter Hettche s'intéresse pour sa part de près à l'amitié épistolaire, emplie d'admiration emphatique, entre Marie von Ebner-Eschenbach et son contemporain Paul Heyse (1830-1914), en qui elle voyait un mentor littéraire ; outre les signes évidents de sympathie et d'admiration réciproques, qui trouvent largement écho dans leurs journaux, leur correspondance et les volumes de nouvelles qu'ils se dédient l'un à l'autre, Walter Hettche met pour la première fois en lumière les similitudes structurelles entre la nouvelle Vroni (1891) de Heyse et Eine Todtenwacht (1892) d'EbnerEschenbach.

5 L'ouvrage se clôt sur trois articles complémentaires qui ont pour objet la " mémoire ", voire la patrimonialisation de Marie von Ebner-Eschenbach. Dans le premier, Kyra Waldner aborde le vaste problème posé par la dispersion du Nachlass de l'auteure. Dans le deuxième, Ingeborg Schemper et Caroline Mang décrivent les monuments qui ont été élevés à la gloire de l'auteure et qui témoignent, chez elle, d'un tiraillement entre un souci de modestie et un désir de représentation conforme à son rang social et littéraire. Dans le troisième, Ulrike Tanzer fait un état des lieux précis de la recherche sur l'œuvre de Marie von Ebner-Eschenbach: par rapport à un certain nombre de ses contemporains comme Theodor Storm, Gottfried Keller ou Johann Nestroy, sa réception apparaît sensiblement en retrait, aussi bien en termes de soutiens institutionnels que de présence éditoriale. L'achèvement en 2015 d'une édition en quatre volumes des œuvres de Marie von Ebner-Eschenbach parue au Residenz-Verlag (sous la direction d'Evelyne Polt-Heinzl, de Daniela Strigl et d'Ulrike Tanzer), la parution dans cette même maison d'édition de la biographie de l'auteure par Daniela Strigl (Berühmt sein ist nichts: Marie von Ebner-Eschenbach. Eine Biographie, 2016), ainsi que la publication de la correspondance entre Marie von Ebner-Eschenbach et Josephine von Knorr chez De Gruyter en 2016 (sous la direction de Ulrike Tanzer, Irene Fußl, Lina Maria Zangerl et Gabriele Radecke), sans oublier la tenue de deux colloques internationaux consacrés à l'auteure, permettent toutefois d'envisager des jours meilleurs.

6 Cet ouvrage collectif, tout en dressant un bilan utile de la recherche actuelle sur l'œuvre de Marie von Ebner-Eschenbach, ouvre un certain nombre de perspectives (voir à ce sujet les pistes de réflexion proposées par Ulrike Tanzer, p. 225-228) qui ne manqueront pas, à l'avenir, de s'avérer fructueuses pour quiconque souhaite 
(re)découvrir aujourd'hui la complexité et les ambivalences d'une auteure phare de la littérature autrichienne du XIX et du début du XXe siècle. 